\title{
THE IMPACT OF AN EVENT-TRIGGERED VIDEO INTERVENTION ON RURAL TEENAGE DRIVING
}

\author{
Daniel V. McGehee, Cher Carney, Mireille Raby, \\ John D. Lee, and Michelle L. Reyes \\ University of Iowa \\ Iowa City, Iowa, USA \\ E-mail: daniel-mcgehee@uiowa.edu
}

\begin{abstract}
Summary: This study examines the ability of an event-triggered video system to extend parental involvement into the independent driving phase of newly licensed teen drivers. The system provides both immediate feedback and a 20 -second video clip, giving the teen driver and their parent the opportunity to review and learn from their mistakes as well as good responses. The event-triggered video system was placed in the vehicles of 25 teen drivers (ages 16-17) for 57 weeks. The first nine weeks established a within-subject baseline; no parental or system feedback was given during this time. During the next 40 weeks, feedback was provided to the teen driver in the form of a blinking LED on the camera and a weekly report card mailed to the parents. The report showed the driver's weekly and cumulative performance regarding unsafe behaviors and seatbelt use relative to the other participants. The last eight weeks was a second baseline period. Results revealed two distinct groups: one that triggered few events and one that triggered many events. Combining this emerging technology with parental weekly review of safety-relevant incidents resulted in a significant and lasting decrease in events for most of the teens that triggered many events.
\end{abstract}

\section{INTRODUCTION}

Motor vehicle crashes are the leading cause of death and injury among American teenagers, resulting in more than 3,000 deaths per year (Mayhew et al., 2003; McCartt, Shabanova, \& Leaf, 2003; Shope, 2006). According to the Fatality Facts 2005 on Teenagers, published by the Insurance Institute for Highway Safety, 5,288 teenagers (13-19 years old) died in 2005 in the United States from motor vehicle crashes (IIHS, 2007). This number represents more than a third of all deaths of 16- to 18-year-olds (Chen, Baker, Braver \& Li, 2000; IIHS, 2004). In fact, IIHS (2007) reported that 40 percent of all deaths from all causes in 2000 for 16- to 19-year-old teenagers were related to motor vehicle crashes.

Sixteen-year-olds have the highest crash rates of all (Mayhew, Simpson \& Pak, 2003; Williams, 2003). The tendency for teenage drivers to have high crash rates (fatal and nonfatal), compared with drivers of all ages, remains true no matter how one analyzes the data (i.e., whether crash rates are based on the total number of teenagers, on the number of licensed teens, or on miles driven (IIHS, 2007)). In fact, because licensure rates and miles driven per license holder are generally lower among 16-19-year-olds, their fatality rates per mile driven tend to be even more extreme compared to older drivers (IIHS, 2007). 
The mechanisms that underlie the crashes of young drivers are difficult to isolate. This difficulty stems from the natural confounding of age, lifestyle, and experience, but it also stems from the composite nature of the multi-level control task that is driving (Summala, 1996). Failures of control at each level and the subsequent propagation of these failures across levels describe many of the factors that contribute to the overrepresentation of young drivers in car crashes (Lee, 2007). Specific causes include imperfectly learned vehicle control skills (Patten, Kircher, Ostlund, Nilsson, \& Svenson, 2006; Shinar, Meir, \& Ben-Shoham, 1998); poor ability to anticipate and identify hazards (Fisher, Pollatsek, \& Pradhan, 2006; McKenna, Horswill, \& Alexander, 2006; Pollatsek, Fisher, \& Pradhan, 2006); willingness to take risks, such as shorter following distances and higher speeds (Evans \& Wasielewski, 1983; Laapotti, Keskinen, Hatakka, \& Katila, 2001); poor calibration of abilities relative to driving demands (Horswill, Waylen, \& Tofield, 2004; Ivancic \& Hesketh, 2000; Matthews \& Moran, 1986); and sensitivity to peer influences in adopting inappropriate norms (Lin \& Fearn, 2003; Simons-Morton, Lerner, \& Singer, 2005).

A variety of technologies are now becoming more available to potentially mitigate teen driving risk (Lee, 2007). Such technologies and interventions show promise for improving driving safety among teen drivers (McGehee, Carney, Raby, Lee \& Reyes, 2007; Lee, 2007). In addition to technological solutions, parents of teenage drivers also play a significant role in their teen's driving safety. They are responsible for enforcing the graduated driver licensing policies and driving restrictions by controlling access to their teen's vehicle (Simons-Morton, Hartos, \& Beck, 2003). Even a brief intervention at the time of teen provisional licensure has proven successful in increasing parental restrictions on teen driving privileges (Simons-Morton et al, 2003). By simply showing a short video on the risks of teen driving and providing both parents and teens with a driving agreement listing teen driving risks and suggestions for families on setting driving rules for teens, Simons-Morton and colleagues (2003) reported significant treatment group differences that were still present nine months later. A recent literature review supports this notion, indicating that parental involvement in management of novice teen driving positively affects teen driver safety (Simons-Morton \& Ouimet, 2006). Therefore, a working hypothesis for this research is that direct feedback regarding potentially unsafe driving behaviors, along with parent-teen interaction regarding these behaviors, will reduce the number of safety-relevant errors made by teen drivers.

\section{METHODS}

\section{Participants}

Twenty-five 16-17 year old drivers (13 females and 12 males), who had obtained their driver's license in the past 6 to 12 months, were recruited from a small rural high school in Tiffin, Iowa. The school district covers a 162-square-mile radius in which nearly all driving occurs on rural highways and gravel roads. Prior to induction into the study, the subjects' driving experience ranged from 3 months to 1 year. 


\section{Data collection}

Data collection began in March 2006 and data were first collected for a nine-week period to establish a baseline estimate of driver behavior. No feedback was provided during the baseline period.

During the next forty weeks, teens were provided with two types of feedback. The first was realtime and consisted of an LED on the recording unit blinking immediately after an event was triggered. This informed the driver that the maneuver just completed exceeded the safety limits defined by lateral and longitudinal acceleration thresholds. The second type of feedback was a weekly report card accompanied by a CD containing all safety-relevant video clips for that week. The report showed the driver's weekly and cumulative performance regarding unsafe behaviors and seatbelt use relative to the other participants.

We supplied proprietary software for viewing the CDs that helped ensure confidentiality by only functioning on the host computer. Parents were encouraged to review the recorded clips and weekly report card with their teen each week.

During the final eight weeks of the project, no feedback was provided. This second baseline phase assessed whether the effect of the feedback persisted and led to a lasting change in the teens' driving behavior.

\section{Apparatus}

Each participant's vehicle was equipped with an event-triggered video recording system made by DriveCam. This system is a palm-sized device that integrates two video cameras (forward and interior view), a two-axis accelerometer, and a wireless transmitter. Video data is continuously buffered 24 hours/day but only writes to internal memory when an acceleration threshold is exceeded. DriveCam uses thresholds that roughly correspond to g-forces (+/- 10 percent). These thresholds refer to accelerometer readings that reflect changes in vehicle velocity or the lateral forces acting on the vehicle when cornering. If the acceleration exceeds the threshold value, then an event is triggered. The trigger thresholds for this research project were 0.55 lateral and 0.50 longitudinal. Each video clip captures the 10 seconds preceding and the 10 seconds following the threshold exceedance. The DriveCam can also be manually activated to record a video clip. Throughout the entire study, the teens were asked to manually activate the camera and provide a weekly odometer reading. All data were automatically downloaded from the device via a secure wireless network whenever the participant parked in the high school parking lot. Once downloaded, encrypted data were compiled for coding.

\section{Analyses}

Members of the research team reviewed all video clips. Any video data captured while a nonconsented driver was using a participant's vehicle were deleted and not viewed. False triggers (e.g. hitting a pothole) were tabulated separately and were accompanied with a brief narrative describing what caused the trigger. In some cases, false triggers revealed safety-relevant behavior (e.g. driver talking on a cell phone when the vehicle hit the pothole). This information 
was included in the weekly report sent to parents. Finally, all "true" triggers were analyzed and coded for the nature of the event (e.g., trigger type and strength); incident type (e.g., following too closely); environmental conditions (e.g., wet roadway); road type and geometry (e.g., straight and 2-lane highway); contributing factors (e.g., fatigue); and social influences. All 'true' triggers were placed on a CD and mailed to the teen and their parent for review.

\section{RESULTS}

\section{Phase 1 - Baseline (9 weeks)}

During the baseline period, participants drove a total of approximately 44,200 miles, or about 36 miles per day. This primarily reflected their daily commute to and from school. Vehicles may have been occasionally driven by someone else (e.g., parent) but were driven primarily by the participants. Estimates of miles driven were derived from odometer readings.

During this baseline period, analyses revealed that the participants were divided into two groups, one group of 18 drivers (low-frequency group) that averaged 2.8 events per 1000 miles, and another group of six drivers (high-frequency group) that averaged 18.8 events per 1000 miles, more than six times the rate of the first group. One driver who was originally included in the high-frequency group was ultimately excluded from analysis.

\section{Phase II - Intervention (40 weeks)}

During the 40-week intervention, the teen drivers accumulated approximately 275,000 miles (independent of the baseline). Although the intervention phase started towards the end of the school year, participants' daily average mileage remained similar to that of the baseline period, and showed no difference between their summer break (through week 17) and return to school. Compared to the 36 miles per day during the baseline, drivers averaged approximately 40 miles per day during the intervention period.

Of interest is whether safety-relevant events declined after the onset of the intervention and whether the reduction in safety-relevant events during the first nine weeks of intervention was the same for all drivers. Figure 1 shows that the two driver groups reacted differently to the intervention. The 18 low-frequency drivers did not change their behavior significantly_possibly reflecting a floor effect, maintaining an average of approximately 2.0 safety-relevant events per 1000 miles driven, throughout the entire intervention phase. However, the six high-frequency drivers showed a dramatic $72 \%$ reduction, dropping from an average of 18.8 to 5.3 safetyrelevant events per 1000 miles in the first nine weeks of the intervention. After an additional nine weeks of the feedback intervention, the six high-frequency drivers group further dropped their safety-relevant events for a total $86 \%$ decline from the baseline, averaging 2.7 events per 1000 miles. They maintained an average of 2.6 events per 1000 miles throughout the remaining weeks of the intervention, slightly above the low-frequency group. The number of events per 1000 miles were transformed using square root, and mixed model ANOVA for driver group and phase showed a significant interaction between driver group and phase $(F(5,108)=11.4, p<.0001)$, and a significant main effect of group and phase. A similar pattern emerges for the incidents (including near-crashes and crashes), where the six high-frequency drivers benefited the most 
from the intervention, dropping their higher incident rates to almost the level of their lowfrequency peers after 18 weeks of intervention. The interaction between driver group and phase was significant, $F(5,108)=7.93, \mathrm{p}<.0001$.

\section{Phase III - Post-intervention Baseline (8 weeks)}

For the final eight weeks, we removed both the real-time and the weekly feedback. While maturation effects must be considered in any research that evaluates an intervention among populations that are still developing physically and psychologically, this two-month period provides an initial estimate of whether feedback can have a lasting effect on driving behavior.

During this phase, participants logged an estimated 47,300 miles. The high-frequency drivers maintained a low number of safety-relevant events (2.2 events/1000 miles) during this second baseline. The number of events for the low-frequency drivers increased by about $50 \%$ (3.1 events per 1000 miles) compared to the intervention period; however, this increase was not significantly different from any of the intervention periods or the initial baseline period. This increase was mostly driven by one of the 18 low-frequency drivers who averaged over 29 events per 1000 miles in the post-intervention baseline. Another low-frequency driver averaged 7.3 events per 1000 miles while the remaining 16 drivers averaged less than 3.3 events per 1000 miles.

The driver who was excluded from the analysis triggered 50.6 events per 1000 miles in the initial baseline and this fell to an average of 7.5 events during intervention. However, in the postintervention baseline, the driver triggered 126 events, four times more than any other driver, resulting in 46.9 events per 1000 miles.

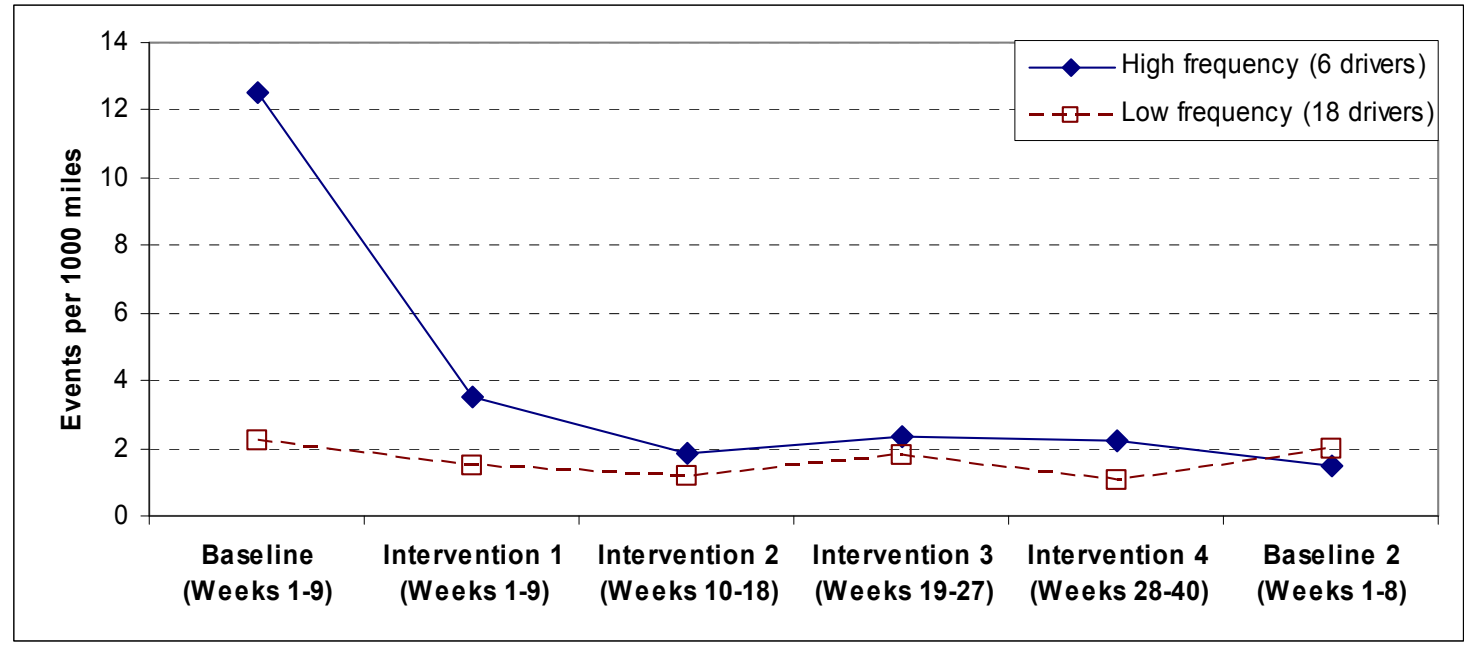

Figure 1. Number of safety-relevant events per 1000 miles for drivers who had high and low event frequency in the initial baseline period

\section{CONCLUSIONS}

This research demonstrates that real-time feedback, paired with a weekly teen and parent review of those events, shows promise. This feedback may help teen drivers, particularly those who 
experience a large number of incidents, become aware of their unsafe driving behaviors and improve their driving. These data suggest an important difference in how the intervention might influence teens. Teens with a high rate of events before receiving feedback showed a dramatic decline in the rate of events. This effect generally persists after the feedback is removed. To the extent that event rates correlate with crash rates, as suggested by Heinrich's triangle (Heinrich et al., 1980), video-based feedback represents a way to reduce crash rates while it is installed in teenagers' cars and may also promote persistent changes.

One promise of the video feedback intervention considered in this study is that it would reduce teen fatalities by helping them learn to drive more safely during their first months of unsupervised driving. One explanation for the reduction in events is that the teens modified their behavior to avoid triggering the system by slowing down for turns, curves and intersections, planning ahead, and looking further down the roadway to allow themselves more time to react to traffic situations. Even if this video feedback only modified behavior when it was installed in the vehicle, it could save thousands of lives. The second promise of this type of intervention is that it might induce long-term changes in driver behavior that would follow the teens into adulthood. A persistent change in driving habits could have a substantial effect on fatalities, as it would ultimately reduce the crash rate of the entire driving population. A multi-year longitudinal study is needed to assess the long-term effects of this intervention.

\section{ACKNOWLEDGMENTS}

The authors wish to thank Ted Loerke, Sam Geraci, and Ryan Rist at American Family Insurance for the generous research grant that funded this project. We also would like to thank Rusty Weiss and DriveCam for their efforts to make this a successful project. Jeff Dolan, Jeff Whitney, Corey Kreutz and Jeff Gordon (University of Iowa NADS) installed and maintained the systems. Finally, we are enormously grateful to the faculty, staff, and students at Clear Creek Amana High School-especially to principal Tom McDonald, and secretaries Jill Dietrich and Deanna Webster.

\section{REFERENCES}

Chen, L-H., Baker, S.P., Braver, E.R., \& Li, G. (2000). Carrying passengers as a risk factor for crashes fatal to 16- and 17-year-old drivers. JAMA, 282(12), 1578-1582.

Evans, L., \& Wasielewski, P. (1983). Risky driving related to driver and vehicle characteristics. Accident Analysis \& Prevention, 15(2), 121-136.

Fisher, D.L., Pollatsek, A.P., \& Pradhan, A. (2006). Can novice drivers be trained to scan for information that will reduce their likelihood of a crash? Injury Prevention, 12, 25-29.

Heinrich, H.W., Petersen, D., Roos, N. (1980). Industrial Accident Prevention. New York, NY: McGraw-Hill.

Horswill, M. S., Waylen, A. E., \& Tofield, M. I. (2004). Drivers' ratings of different components of their own driving skill: A greater illusion of superiority for skills that relate to accident involvement. Journal of Applied Social Psychology, 34(1),177-195.

Insurance Institute for Highway Safety. (2007). Q\&A: Teenagers: General. Available at: http://www.iihs.org/research/qanda/teens.html. Accessed March 17, 2007. 
Ivancic, K., \& Hesketh, B. (2000). Learning from errors in a driving simulation: effects on driving skill and self-confidence. Ergonomics, 43(12), 1966-1984.

Laapotti, S., Keskinen, E., Hatakka, M., \& Katila, A. (2001). Novice drivers' accidents and violations - a failure on higher or lower hierarchical levels of driving behaviour. Accident Analysis and Prevention, 33(6), 759-769.

Lee, J.D. (2007). Technology and the teen driver. Journal of Safety Research, 38, 203-313.

Lin, M. L., \& Fearn, K. T. (2003). The provisional license: nighttime and passenger restrictions a literature review. Journal of Safety Research, 34(1), 51-61.

Matthews, M. L., \& Moran, A. R. (1986). Age differences in male drivers' perception of accident risk: The role of perceived driving ability. Accident Analysis \& Prevention, 18(4), 299-313.

Mayhew, D.R., Simpson, H.M., \& Pak, A. (2003). Changes in collision rates among novice drivers during the first months of driving. Accident Analysis and Prevention, 25, 683-691.

McCartt, A.T., Shabanova, V.I., \& Leaf, W.A. (2003). Driving experience, crashes and traffic citations of teenage beginning drivers. Accident Analysis and Prevention, 35(3), 311-320.

McGehee, D., Carney, C., Raby, M., Lee, J., Reyes, M. (2007). Extending parental mentoring using an event-triggered video intervention in rural teen drivers. Journal of Safety Research, $38,215-227$.

McKenna, F. P., Horswill, M. S., \& Alexander, J. L. (2006). Does anticipation training affect drivers' risk taking? Journal of Experimental Psychology-Applied, 12(1), 1-10.

Patten, C. J. D., Kircher, A., Ostlund, J., Nilsson, L., \& Svenson, O. (2006). Driver experience and cognitive workload in different traffic environments. Accident Analysis and Prevention, $38(5), 887-894$.

Pollatsek, A., Fisher, D. L., \& Pradhan, A. (2006). Identifying and remedying failures of selective attention in younger drivers. Current Directions in Psychological Science, 15(5), 255-259.

Shinar, D., Meir, M., \& Ben-Shoham, I. (1998). How automatic is manual gear shifting? Human Factors, 40(4), 647-654.

Shope, J.T. (2006). Influences on youthful driving behavior and their potential for guiding interventions to reduce crashes. Injury Prevention, 12 (Suppl I), i9-i14.

Simons-Morton, B.G., Hartos, J.L., \& Beck, K.H. (2003). Persistence of effects of a brief intervention on parental restrictions of teen driving privileges. Injury Prevention, 9, 142-146.

Simons-Morton, B.G., Lerner, N., \& Singer, J. (2005). The observed effects of teenage passengers on risky driving behavior of teenage drivers. Accident Analysis \& Prevention, 37, 973-982.

Simons-Morton, B., \& Ouimet, M-C. (2006). Parent involvement in novice teen driving: a review of the literature. Injury Prevention, 12 (Suppl I), i30-i37.

Summala, H. (1996). Accident risk and driver behaviour. Safety Science, 22(1-3), 103-117.

Williams, A.F. (2003). Teenage drivers: patterns of risk. Journal of Safety Research, 34, 5-15. 